\title{
Anti-inflammatory potential of saponins derived from cultured wild ginseng roots in lipopolysaccharide-stimulated RAW 264.7 macrophages
}

\author{
GYEONG-JIN YU ${ }^{1 *}$, IL-WHAN CHOI ${ }^{2 *}$, GI-YOUNG KIM ${ }^{3}$, BYUNG-WOO KIM ${ }^{4,5}$, CHEOL PARK ${ }^{6}$, \\ SU-HYUN HONG ${ }^{1}$, SUNG-KWON MOON ${ }^{7}$, HEE-JAE CHA ${ }^{8}$, YOUNG-CHAE CHANG ${ }^{9}$, \\ KEE YOEUP PAEK ${ }^{10}$, WUN-JAE KIM ${ }^{11}$ and YUNG HYUN CHOI ${ }^{1,4}$
}

\author{
${ }^{1}$ Department of Biochemistry, Dongeui University College of Korean Medicine, Busan 614-052; \\ ${ }^{2}$ Department of Microbiology, College of Medicine, Inje University, Busan 608-756; ${ }^{3}$ Laboratory of Immunobiology, \\ Department of Marine Life Sciences, Jeju National University, Jeju 690-756; ${ }^{4}$ Anti-Aging Research Center and \\ Blue-Bio Industry RIC, ${ }^{5}$ Department of Life Science and Biotechnology and ${ }^{6}$ Department of Molecular Biology, \\ Dongeui University, Busan 614-714; ${ }^{7}$ School of Food Science and Technology, Chung-Ang University, Ansung 456-756; \\ ${ }^{8}$ Department of Parasitology and Genetics, Kosin University College of Medicine, Busan 602-072; \\ ${ }^{9}$ Department of Medicine, Catholic University of Daegu School of Medicine, Daegu 705-718; \\ ${ }^{10}$ Department of Horticultural Science, College of Agriculture, Life and Environmental Sciences and \\ ${ }^{11}$ Department of Urology, College of Medicine, Chungbuk National University, \\ Cheongju, Chungbuk 361-763, Republic of Korea
}

Received October 2, 2014; Accepted March 16, 2015

DOI: $10.3892 /$ ijmm.2015.2165

\begin{abstract}
Ginseng, namely the root of Panax ginseng Meyer, is a well-known traditional medicine that has been used in Asian countries for thousands of years. Ginseng saponins have been shown to exert a variety of prominent pharmacological effects in a number of diseases. The aim of the present study was to identify the anti-inflammatory effects of total saponins extracted from cultured wild ginseng roots (TSWG) on lipopolysaccharide (LPS)-stimulated RAW 264.7 macrophages. An elevated production of nitric oxide (NO) was detected in the RAW 264.7 cells in response to stimulation with LPS, as shown by NO detection assay using Griess reagent. However, pretreatment with TSWG inhibited the production of NO through the suppression of inducible NO synthase gene expression. Furthermore, the LPS-induced gene expression and production of tumor necrosis factor- $\alpha$ (TNF- $\alpha$ ) and interleukin-1 $\beta$ (IL-1 $\beta$ ) were significantly reduced by treatment with TSWG, as shown
\end{abstract}

Correspondence to: Dr Yung Hyun Choi, Department of Biochemistry, Dongeui University College of Korean Medicine, Yangjeong-dong San 45, Busanjin-gu, Busan 614-052, Republic of Korea

E-mail: choiyh@deu.ac.kr

*Contributed equally

Key words: wild ginseng, anti-inflammatory, nuclear factor- $\mathrm{\kappa} \mathrm{B}$, nuclear factor (erythroid-derived 2)-like 2/heme oxygenase-1, mitogenactivated protein kinases by ELISA, and western blot analysis and RT-PCR, respectively. In the LPS-stimulated RAW 264.7 cells, nuclear factor- $\kappa \mathrm{B}$ $(\mathrm{NF}-\kappa \mathrm{B})$ was translocated from the cytosol to the nucleus, while pre-treatment with TSWG induced the sequestration of NF- $\kappa \mathrm{B}$ in the cytosol through the inhibition of the inhibitor of $\kappa \mathrm{B}$ degradation, as shown by immunofluorescence staining. TSWG also contributed to the downregulation of mitogen-activated protein kinases and Akt in the LPS-stimulated RAW 264.7 cells. Additionally, in the TSWG-treated RAW 264.7 cells, we observed the activation of nuclear factor (erythroidderived 2)-like 2 and an increase in heme oxygenase-1 expression; these effects were associated with the inhibition of the generation of reactive oxygen species. The results from the present study indicate that TSWG exerts anti-inflammatory and antioxidant effects, suggesting that TSWG may be an effective therapeutic agent for inflammatory diseases and prevent cellular damage induced by oxidative stress.

\section{Introduction}

Inflammation, acts physiologically to protect normal host function. It is a complex defense response to eliminate foreign pathogens or damaged cells, and to initiate tissue healing involving the activation of various immune cells. Inflammation is mediated by a number of signaling molecules, including pro-inflammatory mediators and cytokines primarily generated through overactivated macrophages $(1,2)$. The induction of pro-inflammatory gene expression in activated macrophages in response to a variety of stimuli, such as lipopolysaccharide (LPS), is mediated by the activation of cellular signaling pathways, such as nuclear factor- $\kappa \mathrm{B}(\mathrm{NF}-\kappa \mathrm{B})$, phosphatidylinositiol 
3-kinase $(\mathrm{PI} 3 \mathrm{~K}) / \mathrm{Akt}$ and mitogen-activated protein kinases (MAPKs) $(3,4)$. NF- $\kappa \mathrm{B}$ is a transcription factor that plays a central role in the regulation of a number of inflammatoryrelated cytotoxic factors, including inducible nitric oxide (NO) synthase (iNOS) and cyclooxygenase-2 (COX-2), and pro-inflammatory cytokines, including tumor necrosis factor- $\alpha$ (TNF- $\alpha$ ) and interleukin-1 $\beta$ (IL-1 $\beta$ ). The numerous pathway components, including PI3K/Akt and MAPKs, such as extracellular signal-regulated kinase (ERK), c-Jun NH2-terminal kinase (JNK) and p38 MAPK play a crucial role in the expression of pro-inflammatory genes during the inflammatory process through the regulation of the nuclear translocation of $N F-\kappa B(5,6)$. Therefore, treatments aimed at inhibiting the NF- $\kappa$ B, PI3K/Akt and MAPK signaling pathways may be a feasible approach in the treatment of certain inflammatory diseases.

Several lines of evidence have revealed the participation of reactive oxygen species (ROS) in inflammation. Under physiological conditions, ROS are formed as a natural byproduct of the normal metabolism of oxygen and play important roles in cell signaling and homeostasis. However, the excessive production of ROS, which may trigger a cascade of radical reactions, causes oxidative damage to cellular macromolecules, thereby resulting in significant damage to cell structures $(4,7)$. During oxidative stress, various antioxidants and cytoprotective proteins, termed phase-2 detoxification enzymes, provide an anti-oxidant defense mechanism against oxidative stress-induced cellular damage. Heme oxygenase-1 (HO-1) is a representative phase- 2 detoxification enzyme with antiinflammatory, antioxidant and cytoprotective functions that are regulated by the activation of nuclear factor (erythroid-derived 2)-like 2 (Nrf2), which interacts with the antioxidant response element (ARE) (8-10). A wide variety of natural compounds have been shown to activate Nrf2 and its downstream target genes, such as HO-1 for cytoprotection against ROS generation and cellular damage $(11,12)$. Thus, ROS, as well as Nrf2/ HO-1 signaling may be an interesting target for the prevention or therapy of inflammation-related diseases.

Ginseng, the root of Panax ginseng C.A. Meyer, is a wellknown medicinal herb in used in traditional Asian medicine for over 2,000 years. It is held in high regard for its tonic properties. Ginseng has been reported to have a variety of biological properties, including antioxidant, anti-inflammatory, anti-allergic, anti-diabetic, immunomodulatory and antitumor properties, as well as cardiovascular and neuroprotective properties (13-21). Accumulating evidence has indicated that most of the pharmacological functions of ginseng are attributed to a variety of ginsenosides, which are triterpenoid saponins (22). Wild ginseng is not field-cultivated domestically, but grows naturally and is harvested from wherever is found. Commonly, cultivated ginseng is systematically farmed on open land and harvested after a 4-6-year cultivation period. On the other hand, wild ginseng grows in the wild and deep in the mountains with fluctuating daily temperatures and minor exposure to direct sunlight and is very difficult to excavate. It is widely accepted that differences in environmental exposure may result in a variation of the bioactive compounds present in wild ginseng, known to be pharmacologically more effective than field-cultivated ginseng. For example, it has been shown that the concentrations of saponins found in wild ginseng are gener- ally 2-6-fold higher than those of field-cultivated ginseng (23). Moreover, previous studies have indicated that wild ginseng extract enhances the host defense, and has more prominent anti-inflammatory and anticancer activities than cultivated ginseng (24,25). Additionally, wild ginseng may be more effective as an anxiolytic and anti-depressant than cultivated ginseng (26). However, wild ginseng is dilatory in growth and is more sensitive to environmental changes than cultivated ginseng. The resultant low yields and high costs hamper the efforts to meet the increasing market demand, and thus the use of wild ginseng in traditional medicine is very limited. On the other hand, cultured wild ginseng roots, the substitute for natural wild ginseng, are easily obtained by a plant cell culture technique. Cultured wild ginseng has been shown to contain similar or higher levels of ginsenosides than those found in cultivated ginseng, but in different ginsenoside ratios $(27,28)$.

In this study, the anti-inflammatory properties of total saponins extracted from cultured wild ginseng roots (TSWG) were investigated. We examined the effects of TSWG on the production of NO and pro-inflammatory cytokines, such as TNF- $\alpha$ and IL-1 $\beta$, in LPS-stimulated mouse RAW 264.7 macrophages. We also determined whether there is a link between the activation of the NF- $\mathrm{KB}$ and MAPK signaling pathways and the anti-inflammatory properties of TSWG. Additionally, we investigated whether TSWG has any effect on the LPS-induced generation of ROS and the Nrf2/HO-1 signaling pathway.

\section{Materials and methods}

Preparation of TSWG. The tissue cultured wild ginseng adventitious roots were supplied by the Research Center for the Development of Advanced Horticultural Technology, Chunbuk National University (Cheongju, Korea), where large-scale cultures of wild ginseng adventitious roots have been developed. Air-dried roots of cultured wild ginseng were ground to pass through an 80-mesh sieve. The samples were extracted twice with methanol by refluxing at $80^{\circ} \mathrm{C}$ for $2 \mathrm{~h}$, and then the methanol extract was suspended in water and partitioned sequentially with $n$-hexane, chloroform, ethyl acetate and $n$-butanol. Subsequently, the water-saturated $n$-butanol fraction was evaporated to dryness in a vacuum. The recovered crude saponins were loaded onto a Diaion ${ }^{\circledR}$ HP-20 MCI gel (SigmaAldrich Chemical Co., St. Louis, MO, USA), and the sugar residues were then removed with $40 \% \mathrm{CH}_{3} \mathrm{OH}$. The fractions were eluted with $60-80 \% \mathrm{CH}_{3} \mathrm{OH}$, collected and then dried to obtain TSWG. TSWG was diluted with the medium, to the desired concentration prior to use.

Cell culture and MTT assay. RAW 264.7 murine macrophagelike cells were purchased from American Tissue Culture Collection (ATCC, Manassas, VA, USA). The cells were grown in Dulbecco's modified Eagle's medium (DMEM) supplemented with $10 \%$ fetal bovine serum (FBS) and $1 \%(\mathrm{v} / \mathrm{v})$ penicillin $(100 \mathrm{U} / \mathrm{ml}) /$ streptomycin $(100 \mu \mathrm{g} / \mathrm{ml})$ under a humidified condition of $5 \% \mathrm{CO}_{2}$ at $37^{\circ} \mathrm{C}$. An inflammatory response was induced in the RAW 264.7 cells by LPS (purchased from Sigma-Aldrich Chemical Co.). DMEM, FBS and penicillin/streptomycin were purchased from Gibco-BRL (Grand Island, NY, USA). Cell viability was measured based on the formation of blue formazan that is metabolized from 
colorless 3-(4,5-dimethylthiazol-2-yl)-2,5-diphenyltetrazolium bromide (MTT; Sigma-Aldrich Chemical Co.) by mitochondrial dehydrogenases, which are active only in live cells. Briefly, the RAW 264.7 cells $\left(5 \times 10^{5}\right.$ cells $\left./ \mathrm{ml}\right)$ were seeded in a 96-well plate. The cells were pre-treated with various concentrations of TSWG for $1 \mathrm{~h}$ and then stimulated with $100 \mathrm{ng} / \mathrm{ml}$ LPS for $24 \mathrm{~h}$. Following incubation with TSWG and LPS, the cultured medium was changed to fresh medium and the cells were incubated with $0.5 \mathrm{mg} / \mathrm{ml}$ MTT solution for $3 \mathrm{~h}$. The supernatant was discarded and the formazan blue, which was formed in the cells, was dissolved with dimethyl sulfoxide (DMSO). The optical density was measured at $540 \mathrm{~nm}$ using an enzyme-linked immunosorbent assay (ELISA) plate reader (Dynatech Laboratories, Chantilly, VA, USA).

Measurement of NO production. The concentrations of NO in the culture supernatants were determined by measuring the levels of nitrite, which is a major stable product of NO, using Griess reagent (Sigma-Aldrich Chemical Co.). For this purpose, the RAW 264.7 cells were seeded in each well of a 96-well plate. The cells were pre-treated with the indicated concentrations of TSWG for $1 \mathrm{~h}$ and then stimulated with $100 \mathrm{ng} / \mathrm{ml}$ LPS. Following incubation for $24 \mathrm{~h}$, the supernatant of each well was mixed with the same volume of Griess reagent, for $10 \mathrm{~min}$ at room temperature in the dark. Nitrite levels were determined using an ELISA plate reader at $540 \mathrm{~nm}$, and nitrite concentrations were calculated by referencing a standard curve generated with known concentrations of sodium nitrite.

Measurement of TNF- $\alpha$ and IL-1 $\beta$ production. The inhibitory effects of TSWG on the production of TNF- $\alpha$ and IL- $1 \beta$ were examined using ELISA kits (R\&D Systems Inc., Minneapolis, MN, USA). The cell culture conditions were the same as those used for the nitrite measurement assay. Following incubation with TSWG and LPS for $24 \mathrm{~h}$, the concentrations of TNF- $\alpha$ and IL-1 $\beta$ in the culture medium were determined using a selective ELISA kit, according to the manufacturer's instructions.

Reverse transcription-polymerase chain reaction (RT-PCR). The cells were either incubated with TSWG $(400 \mu \mathrm{g} / \mathrm{ml})$ alone for $24 \mathrm{~h}$, or pre-treated with the indicated concentrations of TSWG for $1 \mathrm{~h}$ prior to stimulation with LPS for $24 \mathrm{~h}$. Total RNA from the cultured cells was isolated using TRIzol reagent (Invitrogen, Carlsbad, CA, USA). cDNA was synthesized from $1 \mu \mathrm{g}$ of total RNA, using AccuPower ${ }^{\circledR}$ RT PreMix (Bioneer, Daejeon, Korea) containing moloney murine leukemia virus reverse transcriptase. iNOS, IL-1 $\beta$ and TNF- $\alpha$ genes were amplified from the cDNA by PCR. A sample of each amplified product was subjected to $1.0 \%$ agarose gel electrophoresis and stained with ethidium bromide (EtBr, Sigma-Aldrich Chemical Co.) and visualized using ultraviolet (UV) illumination. The glyceraldehyde 3-phosphate dehydrogenase (GAPDH) housekeeping gene transcript was used as a control. The primers used for PCR were as follows: iNOS forward, 5'-ATG TCC GAA GCA AAC ATC AC-3' and reverse, 5'-TAA TGT CCA GGA AGT AGG TG-3'; IL-1 $\beta$ forward, 5'-GGG CTG CTT CCA AAC CTT TG-3' and reverse, 5'-GCT TGG GAT CCA CAC TCT CC-3'; TNF- $\alpha$ forward, 5'-TCT CAT CAG TTC TAT GGC CC-3' and reverse, 5'-GGG AGT AGA CAA GGT ACA AC-3'; and GAPDH forward, 5'-AGG CCG

\section{GTG CTG AGT ATG TC-3' and reverse, 5'-TGC CTG CTT CAC CAC CTT CT-3'.}

Protein extraction and western blot analysis. For total protein extraction, the cells were lysed in lysis buffer $[25 \mathrm{mM}$ Tris-Cl (pH 7.5), $250 \mathrm{mM} \mathrm{NaCl}, 5 \mathrm{mM}$ ethylenediaminetetraacetic acid (EDTA), 1\% NP-40, $1 \mathrm{mM}$ phenylmethylsulfonyl fluoride (PMSF) and $5 \mathrm{mM}$ dithiothreitol (DTT)] for $1 \mathrm{~h}$. The insoluble materials were discarded by centrifugation at $13,000 \mathrm{x} \mathrm{g}$ for $20 \mathrm{~min}$ at $4^{\circ} \mathrm{C}$. In a parallel experiment, nuclear and cytosolic proteins were prepared using nuclear extraction reagents (Pierce, Rockford, IL, USA) according to the manufacturer's instructions. The protein concentration of the cell lysate was determined using detergent-compatible protein assay obtained from Bio-Rad (Hercules, CA, USA). Equal amounts of protein were separated by sodium dodecyl sulfate-polyacrylamide gel electrophoresis (SDS-PAGE). The separated protein was transferred onto nitrocellulose membranes (Schleicher \& Schuell BioScience, Inc., Keene, NH, USA) and subsequently blocked with Tris-buffered saline ( $10 \mathrm{mM}$ Tris- $\mathrm{Cl}, \mathrm{pH}$ 7.4) containing $0.5 \%$ Tween-20 and 5\% non-fat dry milk for $1 \mathrm{~h}$ at room temperature. The proteins were probed with primary antibodies at $4^{\circ} \mathrm{C}$ overnight. After probing with the primary antibodies (Table I), the membranes were incubated with horseradish peroxidaseconjugated anti-rabbit immunoglobulin G (IgG; sc-2004, 1:1,000), anti-mouse IgG (sc-2005, 1:1,500) and anti-goat $\mathrm{IgG}$ (sc-2350, 1:1,500) as secondary antibodies (Santa Cruz Biotechnology, Inc., Santa Cruz, CA, USA). Immunoreactive bands were detected using the enhanced chemiluminescence (ECL) detection system (Amersham Corp.) and exposed to X-ray film. Primary antibodies were purchased from Santa Cruz Biotechnology, Inc., Cell Signaling Technology, Inc. (Danvers, MA, USA) and Abcam (Cambridge, UK) (Table I). Lamin B and actin were used as internal controls for the nuclear and cytosolic fractions, respectively.

Immunofluorescence staining. For the detection of the translocation of NF- $\mathrm{kB}$ p65, the RAW 264.7 cells were grown on glass coverslips for $24 \mathrm{~h}$. The cells were pre-treated with $400 \mu \mathrm{g} / \mathrm{ml} \mathrm{TSWG}$ for $30 \mathrm{~min}$ prior to stimulation with $100 \mathrm{ng} /$ $\mathrm{ml}$ LPS. Following incubation for $30 \mathrm{~min}$, the cells were fixed with $3.7 \%$ paraformaldehyde, treated with $0.2 \%$ Triton X-100 and blocked with $2 \%$ bovine serum albumin (BSA). The cells were then sequentially incubated with anti-NF- $\mathrm{B}$ p 65 antibody (Table I), fluorescein isothiocyanate-conjugated donkey anti-rabbit IgG (1:500; \#111-095-003, Jackson ImmunoResearch Laboratories, Inc., West Grove, PA, USA) and 4,6-diamidino2-phenylindole (DAPI; Sigma-Aldrich Chemical Co.) solution. They were then examined under a fluorescence microscope (Carl Zeiss, Jena, Germany).

Measurement of intracellular ROS generation. The intracellular accumulation of ROS was determined using the fluorescent probe, 2',7'-dichlorodihydrofluorescein diacetate $\left(\mathrm{H}_{2}\right.$ DCFDA; Sigma-Aldrich Chemical Co.). Briefly, the RAW 264.7 cells were pre-treated with $400 \mu \mathrm{g} / \mathrm{ml}$ TSWG for $30 \mathrm{~min}$ prior to stimulation with $100 \mathrm{ng} / \mathrm{ml}$ LPS for $30 \mathrm{~min}$. The cells were then incubated for $4 \mathrm{~h}$ at $37^{\circ} \mathrm{C}$ in phosphatebuffered saline (PBS) containing $20 \mathrm{mM}$ H2DCFDA to label the intracellular ROS. ROS production in the cells was 
Table I. Antibodies used in the present study.

\begin{tabular}{|c|c|c|c|}
\hline Antibody & Dilution & Product no. & Species of origin and supplier \\
\hline iNOS & $1: 500$ & SC-7271 & Mouse monoclonal, Santa Cruz Biotechnology, Inc. \\
\hline TNF- $\alpha$ & $1: 500$ & \#3707S & rabbit polyclonal, Cell Signaling Technology, Inc. \\
\hline IL-1 $\beta$ & $1: 500$ & SC-7884 & Rabbit polyclonal, Santa Cruz Biotechnology, Inc. \\
\hline $\mathrm{NF}-\kappa \mathrm{B}$ p65 & $1: 500$ & SC-109 & Rabbit polyclonal, Santa Cruz Biotechnology, Inc. \\
\hline $\mathrm{I} \kappa \mathrm{B} \alpha$ & $1: 500$ & SC-371 & Rabbit polyclonal, Santa Cruz Biotechnology, Inc. \\
\hline Akt & $1: 500$ & SC-8312 & Rabbit polyclonal, Santa Cruz Biotechnology, Inc. \\
\hline p-Akt & $1: 500$ & SC-101629 & Rabbit polyclonal, Santa Cruz Biotechnology, Inc. \\
\hline ERK & $1: 1,000$ & SC-154 & Rabbit polyclonal, Santa Cruz Biotechnology, Inc. \\
\hline p-ERK & $1: 500$ & \#9106S & Mouse monoclonal, Cell Signaling Technology, Inc. \\
\hline p38 & $1: 1,000$ & $\mathrm{SC}-535$ & Rabbit polyclonal, Santa Cruz Biotechnology, Inc. \\
\hline p-p38 & $1: 500$ & \#9211S & Rabbit polyclonal, Cell Signaling Technology, Inc. \\
\hline JNK & $1: 1,000$ & \#9252S & Rabbit polyclonal, Cell Signaling Technology, Inc. \\
\hline p-JNK & $1: 500$ & \#9255S & Mouse monoclonal, Cell Signaling Technology, Inc. \\
\hline Nrf2 & $1: 500$ & SC-13032 & Rabbit polyclonal, Santa Cruz Biotechnology, Inc. \\
\hline $\mathrm{p}-\mathrm{Nrf} 2$ & $1: 500$ & ab76026 & Rabbit monoclonal, Abcam, Inc. \\
\hline $\mathrm{HO}-1$ & $1: 500$ & SC-136960 & Mouse monoclonal, Santa Cruz Biotechnology, Inc. \\
\hline Lamin B & $1: 500$ & SC-6216 & Goat polyclonal, Santa Cruz Biotechnology, Inc. \\
\hline$\beta$-actin & $1: 1,000$ & sc-1616 & Goat polyclonal, Santa Cruz Biotechnology, Inc. \\
\hline
\end{tabular}

monitored using a flow cytometer (FACSCalibur; BectonDickinson, San Jose, CA, USA) using CellQuest Pro software, as previously described (29).

Data analysis. The results are expressed as the means \pm standard deviation (SD). Differences in the mean values between groups were analyzed by one-way analysis of variance followed by Dunnett's test. A P-value of $<0.05$ was considered to indicate a statistically significant difference.

\section{Results}

Effect of TSWG on NO production in LPS-stimulated $R A W 264.7$ cells. Treatment of the cells with LPS alone markedly induced NO production compared with the untreated controls, according to the NO detection assay using Griess reagent. However, pre-treatment with TSWG significantly suppressed the levels of NO production in the LPS-stimulated RAW 264.7 microglial cells, in a concentration-dependent manner $(>400 \mu \mathrm{g} / \mathrm{ml})$ (Fig. 1A). We then performed RT-PCR and western blot analysis to determine whether the inhibition of NO production by TSWG in the LPS-stimulated RAW 264.7 cells was associated with decreased levels of iNOS, which produces $\mathrm{NO}$ as a key mediator of inflammation. TSWG did not induce iNOS expression at the mRNA and protein level in the unstimulated RAW 264.7 cells, while stimulation with LPS increased iNOS expression (Fig. 1B and C). However, TSWG induced a significant decrease in iNOS expression in the LPS-stimulated RAW 264.7 cells in a concentration-dependent manner. These results indicated that TSWG downregulated NO production in the LPS-stimulated RAW 264.7 cells by inhibiting iNOS expression. Additionally, TSWG regulated the expression of iNOS at the transcriptional level.
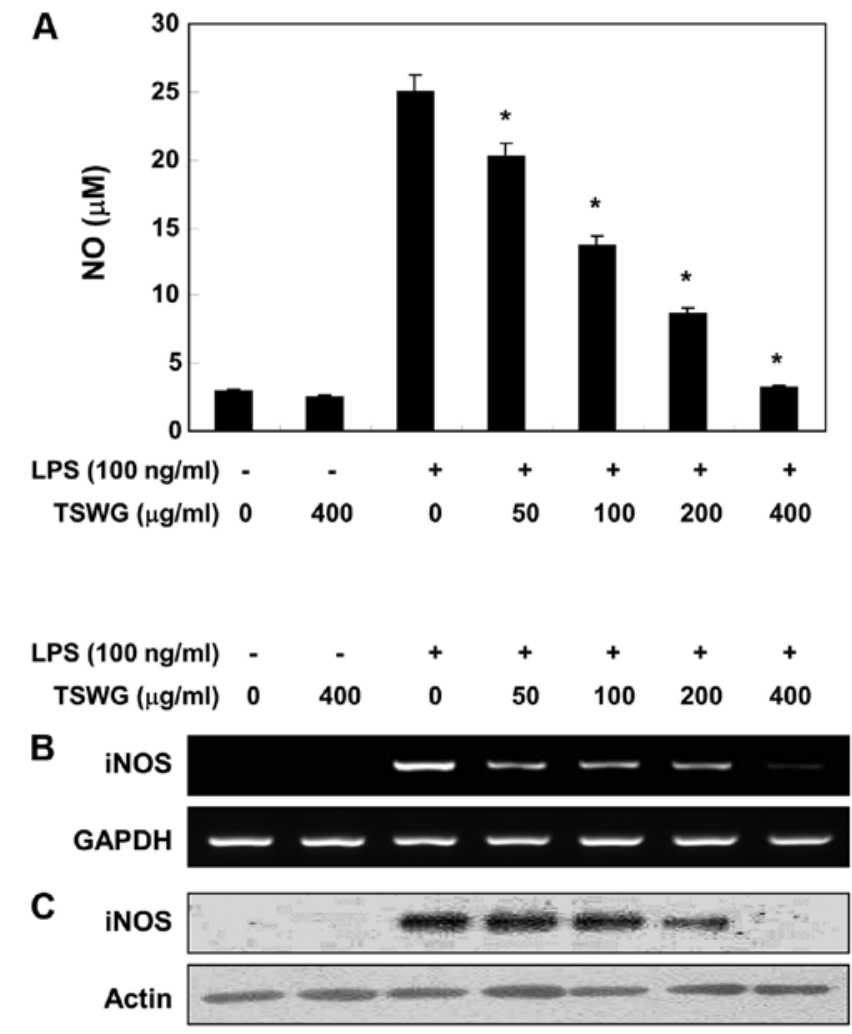

Figure 1. Inhibition of nitric oxide (NO) production and inducible NO synthase (iNOS) expression by total saponins extracted from cultured wild-ginseng roots (TSWG) in lipopolysaccharide (LPS)-stimulated RAW 264.7 cells. RAW 264.7 cells were pre-treated with the indicated concentrations of TSWG for $1 \mathrm{~h}$ and then stimulated with LPS $(100 \mathrm{ng} / \mathrm{ml})$ for $24 \mathrm{~h}$. (A) NO production was measured using Griess reagent. Values are the means \pm SD. ${ }^{*} \mathrm{P}<0.05$ compared with the LPS group. (B and C) The mRNA and protein levels of iNOS were determined by RT-PCR and western blot analysis, respectively. Glyceraldehyde 3-phosphate dehydrogenase (GAPDH) and actin were used as internal controls for RT-PCR and western blot analysis, respectively. 


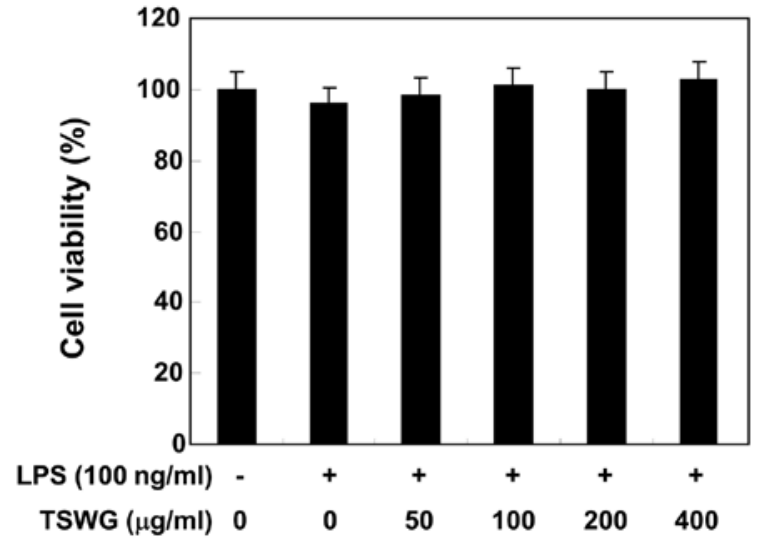

Figure 2. Effects of total saponins extracted from cultured wild-ginseng roots (TSWG) and lipopolysaccharide (LPS) on the viability of RAW 264.7 cells. Cells were treated with the indicated concentrations of TSWG or LPS alone, or pre-treated with TSWG for $1 \mathrm{~h}$ prior to stimulation with LPS; after $24 \mathrm{~h}$, cell viability was assessed by MTT reduction assay. The results are expressed as the percentage of surviving cells over the control cells (without the addition of TSWG). Each value indicates the mean \pm SD of 3 independent experiments.

Cytotoxicity of TSWG. MTT assays were performed using the RAW 264.7 cells treated with TSWG for $24 \mathrm{~h}$ in the presence or absence of LPS (100 ng/ml), to exclude the possibility that the inhibition of NO production was due to cytotoxicity caused by
TSWG. TSWG alone did not affect cell viability at the concentrations used to inhibit NO production (Fig. 2). Furthermore, co-treatment with TSWG and LPS had no cytotoxic effects. These results clearly indicated that the inhibition of NO production in the LPS-stimulated RAW 264.7 cells was not due to the cytotoxic effects of TSWG.

Effect of TSWG on the production of pro-inflammatory cytokines in LPS-stimulated RAW 264.7 cells. We further quantified the production of inflammatory cytokines, such as IL-1 $\beta$ and TNF- $\alpha$ in the culture medium using ELISA to identify the anti-inflammatory properties of TSWG. When the RAW 264.7 cells were treated with TSWG alone, there were no changes observed in the production of IL- $1 \beta$ and TNF- $\alpha$. The production of IL- $1 \beta$ and TNF- $\alpha$ was markedly increased by LPS stimulation; however, pre-treatment with TSWG significantly decreased the production of cytokines in a concentration-dependent manner (Fig. 3A and B). Since cytokine secretion was reduced by treatment wit hTSWG, we evaluated whether the inhibitory effects of TSWG on TNF- $\alpha$ and IL-1 $\beta$ expression are associated with the inhibitory effects on the release of TNF- $\alpha$ and IL-1 $\beta$. Our results indicated that the LPS-induced increase in the TNF- $\alpha$ and IL-1 $\beta$ mRNA levels was reversed by TSWG in a concentration-dependent manner (Fig. 3C). In a parallel experiment, the elevated protein levels of TNF- $\alpha$ and IL-1 $\beta$ resulting from stimultion with LPS
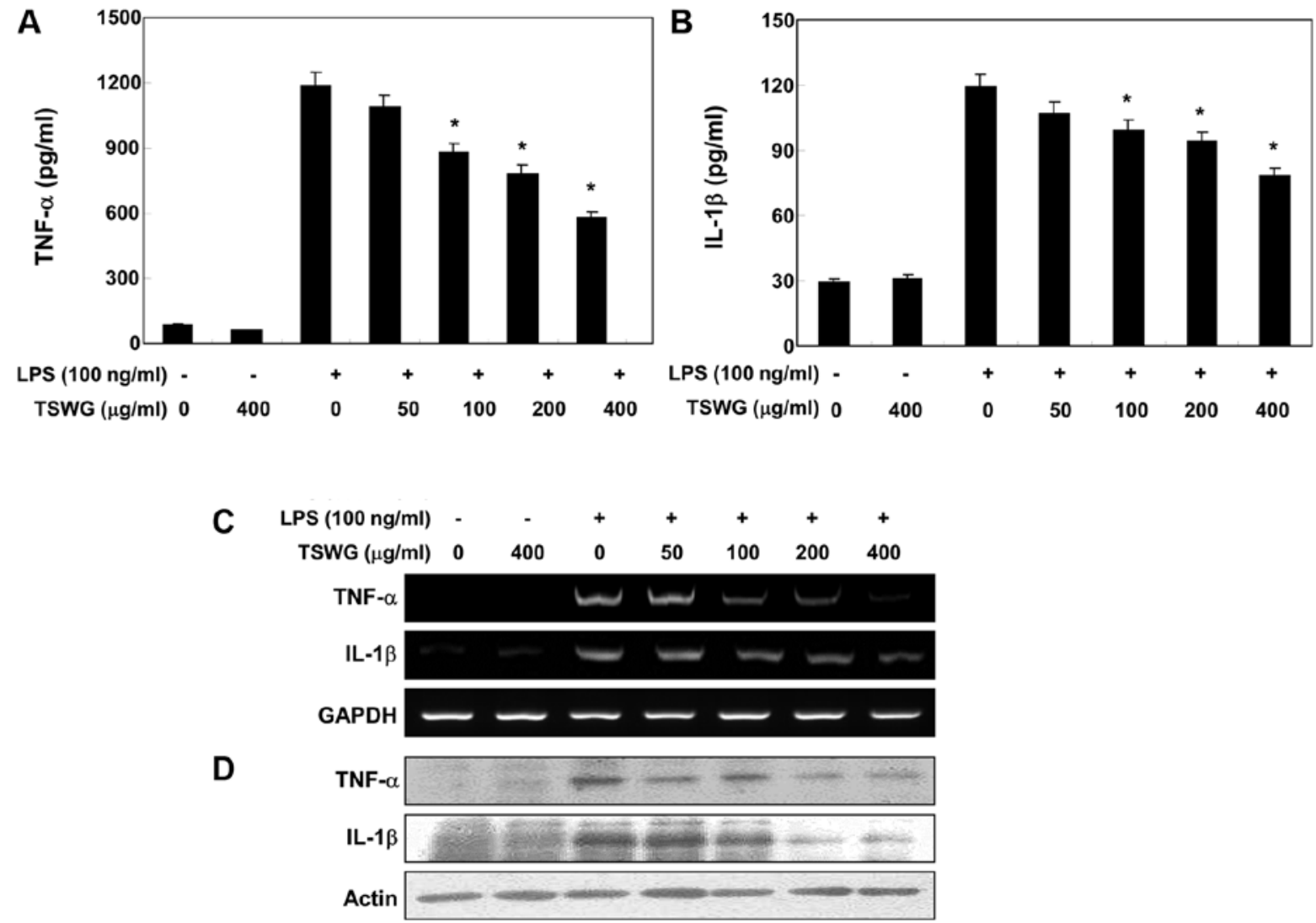

Figure 3. Inhibition of lipopolysaccharide (LPS)-induced tumor necrosis factor- $\alpha$ (TNF- $\alpha$ ) and interleukin-1 $\beta$ (IL-1 $\beta$ ) production and expression by total saponins extracted from cultured wild-ginseng roots (TSWG) in LPS-stimulated RAW 264.7 cells. RAW 264.7 cells were pre-treated with various concentrations of TSWG for $1 \mathrm{~h}$ and then stimulated with LPS (100 ng/ml) for $24 \mathrm{~h}$. (A and B) The production of TNF- $\alpha$ and IL-1 $\beta$ was measured using corresponding ELISA kits. Values are the means \pm SD. ${ }^{*} \mathrm{P}<0.05$ compared with the LPS group. (C and D) The mRNA and protein levels of TNF- $\alpha$ and IL-1 $\beta$ were determined by RT-PCR and western blot analysis, respectively. Glyceraldehyde 3-phosphate dehydrogenase (GAPDH) and actin were used as internal controls for RT-PCR and western blot analysis, respectively. 

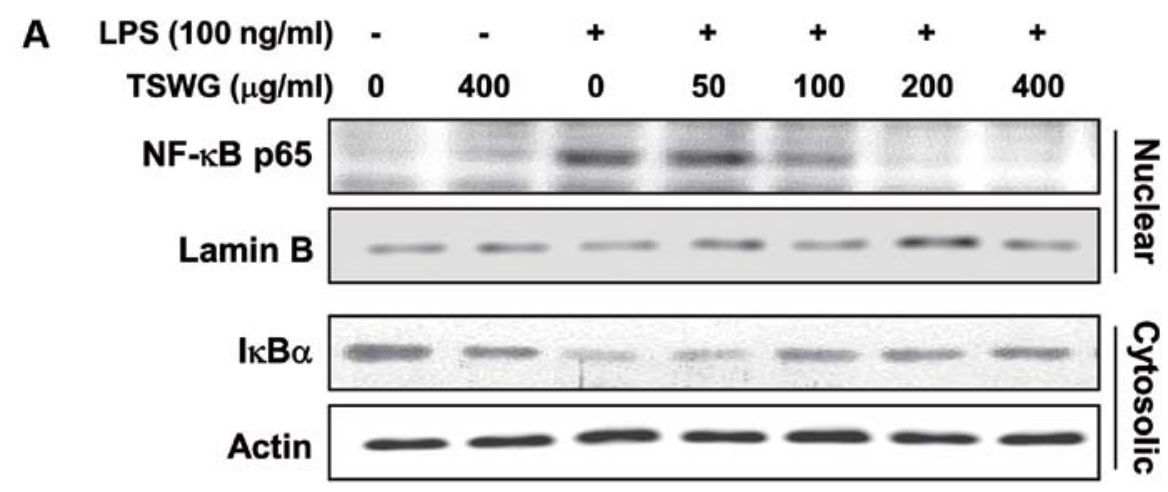

B

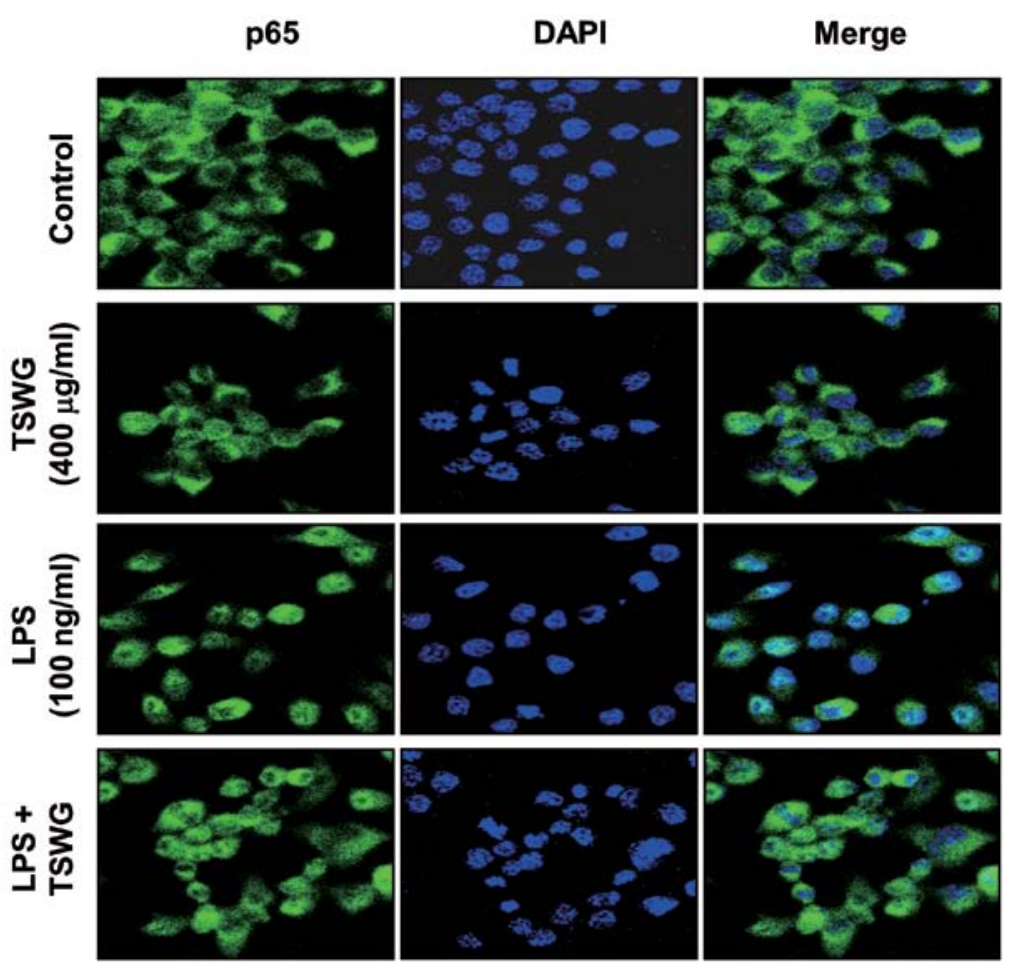

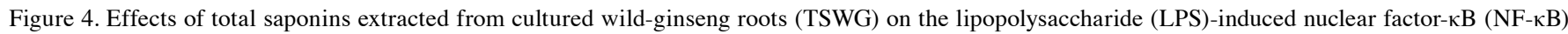
translocation and expression in RAW 264.7 cells. RAW 264.7 cells were pre-treated with the indicated concentrations of TSWG for $1 \mathrm{~h}$ prior to stimulation with

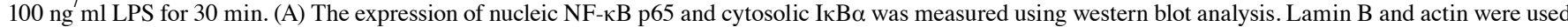
as internal controls for the nuclear and cytosolic fractions, respectively. (B) The localization of NF- $\mathrm{BB}$ p65 was visualized by fluorescence microscopy following immunofluorescence staining with NF-kB p65 antibody (green). In addition, the cells were stained with 4,6-diamidino-2-phenylindole (DAPI) to visualize the nuclei (blue).

were also decreased following treatment with TSWG (Fig. 3D). Our results thus indicate that TSWG negatively regulates the production of pro-inflammatory cytokines, such as IL-1 $\beta$ and $\mathrm{TNF}-\alpha$ at the transcriptional level.

Effect of TSWG on LPS-induced $N F-\kappa B$ translocation in the $R A W 264.7$ cells. Our results revealed that TSWG regulated the expression of iNOS and that of the pro-inflammatory cytokines, TNF- $\alpha$ and IL-1 $\beta$, at the transcriptional level. We then evaluated the expression of nuclear NF- $\mathrm{B}$ p65, a subunit of $\mathrm{NF}-\kappa \mathrm{B}$, and cytosolic I $\kappa \mathrm{B} \alpha$ to further characterize the mechanisms of TSWG-mediated anti-inflammatory responses. We first investigated whof $\mathrm{NF}-\kappa \mathrm{B}$ p65. According to the results of western blot analysis, nuclear NF- $\mathrm{B}$ p65 expression was not specifically increased by TSWG. However, the amount of
$\mathrm{NF}-\kappa \mathrm{B}$ p65 in the nucleus was markedly increased following exposure to LPS for $30 \mathrm{~min}$ (Fig. 4A). However, pre-treatment with TSWG decreased the expression of nuclear NF- $\kappa$ B p65, causing its epxression to return to basal levels. In a parallel experiment, stimulation with LPS decreased $\mathrm{I} \kappa \mathrm{B} \alpha$ expression in the cytosol; however, pre-treatment with TSWG somewhat amplified cytosolic $\mathrm{I} \kappa \mathrm{B} \alpha$ expression in a concentration-dependent manner. We visualized the location of $\mathrm{NF}-\kappa \mathrm{B}$ p65 in the cells by immunofluorescence staining, as experimental evidence of the TSWG-induced NF- $\kappa \mathrm{B}$ inactivation. The cells in the control group and TSWG-treated group showed cytosolic NF- $\kappa$ B p65, while stimulation with LPS facilitated the NF- $\mathrm{B}$ p 65 translocation to the nucleus; however, the cells in the TSWG pre-treated group showed that NF- $\kappa \mathrm{B}$ p65 was arrested in the cytosol (Fig. 4B). These results indicated 


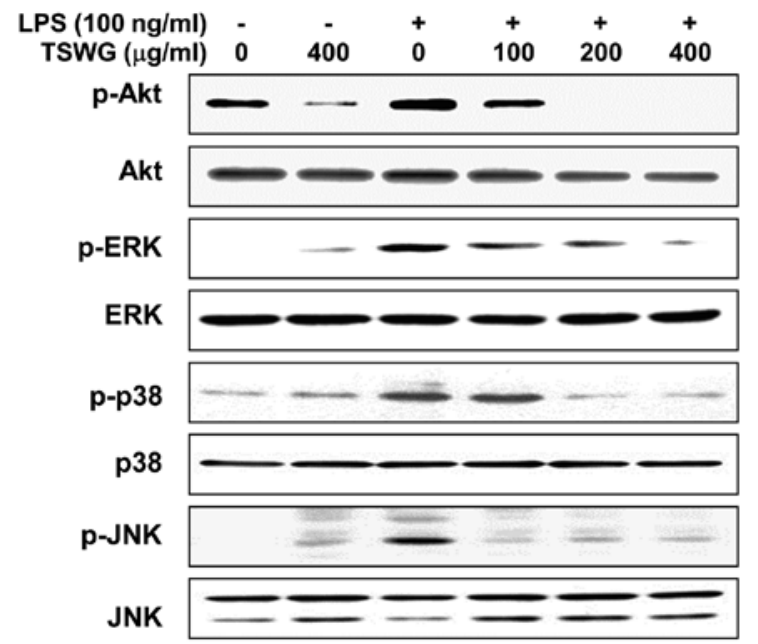

Figure 5. Effects of total saponins extracted from cultured wild-ginseng roots (TSWG) on the activation of Akt and mitogen-activated protein kinases (MAPKs) induced by lipopolysaccharide (LPS) in RAW 264.7 cells. Cells were treated with TSWG $1 \mathrm{~h}$ prior to stimulation with LPS $(100 \mathrm{ng} / \mathrm{ml})$ for $1 \mathrm{~h}$ Total proteins were subjected to $10 \%$ SDS-polyacrylamide gel electrophoresis, followed by western blot analysis using the indicated antibodies.

that treatment with TSWG suppressed NF- $\kappa$ B activity in the LPS-stimulated RAW 264.7 cells by inhibiting the nuclear translocation of NF- $\kappa \mathrm{B}$ and the degradation of $\mathrm{I} \kappa \mathrm{B} \alpha$.

Inhibition of LPS-induced Akt and MAPK activation by TSWG in LPS-stimulated RAW 264.7 cells. Previous studies have demonstrated that the PI3K/Akt and MAPK signaling pathways are involved in the expression of LPS-induced pro-inflammatory genes $(3,4)$. We therefore investigated the effects of TSWG on the LPS-induced activation of Akt and MAPKs, such as ERK, JNK and p38 MAPK by western blot analysis. Stimulation with LPS for $1 \mathrm{~h}$ resulted in a significant increase in the phosphorylated forms of Akt, ERK, JNK and p38 MAPK compared with the control group without altering their unphosphorylated forms (Fig. 5). However, pre-treatment with TSWG for $1 \mathrm{~h}$ attenuated the phosphorylation of Akt and MAPKs in a concentration-dependent manner (Fig. 5). These results suggested that the TSWG-induced inactivation of Akt and MAPKs may cause the suppression of the inflammatory response in LPS-stimulated RAW 264.7 cells.

Suppression of LPS-induced ROS formation by TSWG in $R A W 264.7$ cells. Several studies have reported that the LPS-mediated activation of NF- $\mathrm{NB}$ leads to the enhanced production of ROS as a common second messenger, thereby contributing to the sustained oxidant production during chronic inflammation $(5,6)$. The level of the generation of intracellular ROS was accordingly assessed to determine whether TSWG reduces the level of LPS-induced oxidative stress in RAW 264.7 cells. LPS significantly enhanced ROS production, while pretreatment with TSWG considerably reversed the LPS-induced cellular production of ROS (Fig. 6A). Treatment of the RAW 264.7 cells alone also decreased the ROS levels when compared with the untreated control cells. These results indicated that TSWG was capable of abrogating the LPS-induced increase in ROS production in RAW 264.7 cells.
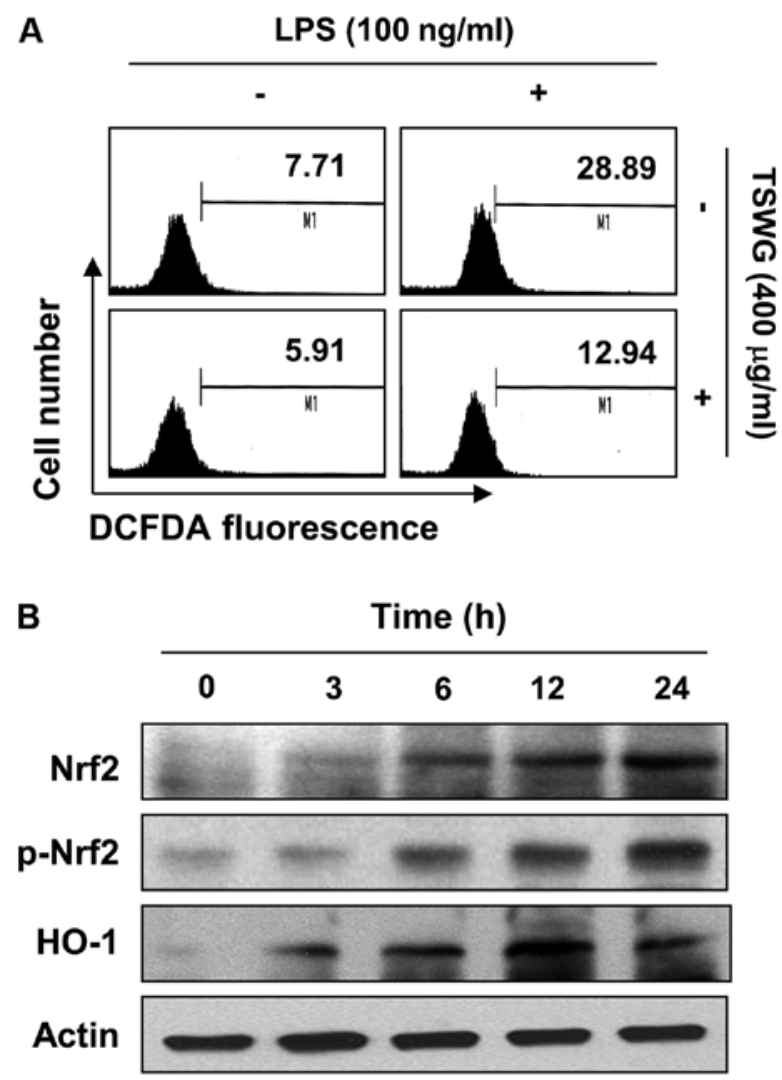

Figure 6. Effects of total saponins extracted from cultured wild-ginseng roots (TSWG) on the lipopolysaccharide (LPS)-induced generation of intracellular reactive oxygen species (ROS), and nuclear factor (erythroid-derived 2)-like 2 (Nrf2) and heme oxygenase-1 (HO-1) expression in RAW 264.7 cells. (A) RAW 264.7 cells were treated with TSWG $(400 \mu \mathrm{g} / \mathrm{ml})$ for $30 \mathrm{~min}$ prior to stimulation with LPS $(100 \mathrm{ng} / \mathrm{ml})$ for $30 \mathrm{~min}$. The cells were incubated with $20 \mathrm{mM} \mathrm{H}{ }_{2}$ DCFDA at $37^{\circ} \mathrm{C}$ for $30 \mathrm{~min}$, and ROS generation was measured using a flow cytometer. Each point represents the mean of 2 independent experiments. (B) RAW 264.7 cells were treated with the indicated concentrations of TSWG for $24 \mathrm{~h}$. The protein expression of Nrf2 and HO-1 was evaluated by western blot analysis. Actin was used as an internal control.

Induction of Nrf-2 and HO-1 expression by TSWG in $R A W 264.7$ cells. Since TSWG prevented the generation of ROS, we hypothesized that pre-treatment with TSWG may facilitate the expression of antioxidant enzymes in the RAW 264.7 cells. We then examined whether the expression of Nrf2 and HO-1 is regulated by TSWG. Following treatment with TSWG, the RAW 264.7 cells showed a gradual increase in the total, as well as in the phosphorylated Nrf2 levels in a time-dependent manner, and this strongly correlated with the increase in HO-1 expression (Fig. 6B). Thee results indicated that TSWG induced HO-1 expression throug the activation of Nrf2.

\section{Discussion}

Macrophage defensive systems play essential roles in teh host defense against bacterial infection by recognition of the threats through specific receptors, particularly the Toll-like receptor 4 (TLR4) complex. However, the enhanced production of inflammatory molecules, including NO, ROS and cytokines by macrophages can cause damage to the host $(30,31)$. In response 
to macrophage activation by LPS, the principal component of the outer membrane of Gram-negative bacteria (32), TLR4 recruits downstream-associated adaptor molecules, including myeloid differentiation factor (MyD88) $(33,34)$. The activation of the MyD88-dependent pathway results in the rapid activation of the $\mathrm{NF}-\kappa \mathrm{B}, \mathrm{PI} 3 \mathrm{~K} / \mathrm{Akt}$ and MAPK pathways, which coordinately regulate inflammation-associated gene activities responsible for host defense. Furthermore, these pro-inflammatory products play key roles in the pathogeneses of various acute and chronic inflammatory diseases $(35,36)$. Therefore, the suppression of the aberrant activation of macrophages may have valuable therapeutic potential for the treatment of various inflammation-associated diseases caused by macrophage activation.

Macrophages have been found to be activated in acute and chronic bacterial inflammatory diseases $(1,2)$. The overproduction of NO in activated macrophages, a potent and critical mediator of inflammatory diseases, is mediated primarily by iNOS, causing tissue injury $(2,37)$. Therefore, the modulation of the iNOS-mediated release of NO from macrophages is considered one of the strategies to develop therapeutic compounds against various inflammatory diseases. In this study, we demonstrated that TSWG exerted its anti-inflammatory effects by suppressing the LPS-induced iNOS mRNA and protein expression, and the subsequent production of NO in RAW 264.7 cells (Fig. 1). These data indicated that the inhibition of NO production in response to TSWG was a result of an inhibition of iNOS gene expression at the transcriptional level. Activated macrophages also exert cytotoxic effects by releasing various pro-inflammatory cytokines, including TNF- $\alpha$ and IL-1 $\beta$ and ROS, that may participate in the inflammatory process $(1,2)$. Moreover, ROS are important contributors to the production of TNF- $\alpha$ and IL- $1 \beta$ in LPS-stimulated macrophages $(38,39)$. In response to LPS, the production of TNF- $\alpha$ and IL-1 $\beta$ was markedly upregulated, but treatment with TSWG significantly inhibited the induction of these cytokines by LPS (Fig. 3A and $\mathrm{B}$ ). In addition, the results from the RT-PCR and western blot analysis revealed that TSWG decreased the TNF- $\alpha$ and IL-1 $\beta$ mRNA levels with a corresponding decrease in the protein levels (Fig. 3C and D). A prerequisite for the proper evaluation of the biological properties of candidate agents is the exact determination of cytotoxicity associated with the prolonged incubation of the cells. In the current study, the concentrations of TSWG used to inhibit the production of $\mathrm{NO}$, TNF- $\alpha$ and IL-1 $\beta$ did not affect cell viability, as measured by MTT assay (Fig. 2), thereby indicating that the anti-inflammatory effects of TSWG on LPS-stimulated RAW 264.7 cells were not simply due to a cytotoxic action of TSWG.

There are several well established highly complex inflammatory signaling pathways. Among these, $\mathrm{NF}-\kappa \mathrm{B}$ is the major transcriptional factor in the production of pro-inflammatory molecules and TLRs are the major pattern recognition receptors to detect conserved microbial products $(40,41)$. In unstimulated macrophages, $\mathrm{NF}-\kappa \mathrm{B}$ is retained in the cytoplasm by $\mathrm{I} \kappa \mathrm{B}$; however, when LPS binds TLR4, I $\mathrm{B}$ is rapidly phosphorylated by the I $\kappa \mathrm{B}$ kinase complex, which results in I $\mathrm{B}$ degradation and the translocation of $N F-\kappa B$ to the nucleus to initiate inflammatory-associated gene expression, including that of iNOS and pro-inflammatory cytokines $(33,39,41)$. Moreover, upon the activation of Akt by PI3K, phosphorylated Akt promotes the activation of $\mathrm{NF}-\kappa \mathrm{B}$ and MAPKs to trigger the transcription of pro-inflammatory regulators in LPS-stimulated macrophages $(5,6,42)$. Thus, suppressing the activation of $\mathrm{NF}-\kappa \mathrm{B}$ by inhibiting I $\kappa \mathrm{B} \alpha$ degradation may be a target for antiinflammatory therapeutic candidates. To investigate whether the inhibition of NF- $\kappa \mathrm{B}$ activation by TSWG was associated with the inactivation of the PI3K/Akt and MAPK pathways, the effects of TSWG on the LPS-induced nuclear translocation of $\mathrm{NF}-\kappa \mathrm{B}$ and the degradation of $\mathrm{I} \kappa \mathrm{B}$ were assessed. The results revealed that TSWG significantly attenuated the LPS-induced $\mathrm{I} \kappa \mathrm{B}$ degradation and the nuclear translocation of $\mathrm{NF}-\kappa \mathrm{B}$ p65 in the LPS-stimulated RAW 264.7 macrophages (Fig. 4). Our results study also demonstrated that pre-treatment with TSWG significantly suppressed the phosphorylation of MAPKs, including ERK, JNK and p38 MAPK, as well as that of Akt in a concentration-dependent manner (Fig. 5). Taken together, these results indicated that the inhibition of NF- $\kappa \mathrm{B}$ activity by TSWG was mediated through the inactivation of PI3K/Akt and MAPKs by the deficiencies in the phosphorylation of Akt or MAPKs. This may lead to the inhibition of pro-inflammatory mediators and cytokine expression in LPS-stimulated macrophages.

HO-1 is one of the phase II enzymes, which are protective proteins expressed to preserve cells against oxidative stress and inflammation. The de novo induction of this protein is mainly due to transcriptional activation mediated by Nrf2 through its interaction with ARE, a regulatory sequence for the transcriptional activation of genes encoding various antioxidant enzymes and phase II detoxifying enzymes. Moreover, it has been shown that HO-1 suppresses the production of proinflammatory mediators, including iNOS, as well as that of cytokines in activated macrophages $(8,10-12)$. Therefore, the activation of the Nrf2/HO-1 pathway is a promising target for the treatment of inflammatory, as well as oxidative stress-mediated diseases. Our results indicated that TSWG induced HO-1 expression in a concentration-dependent manner, which was associated with the activation of Nrf2 (Fig. 6B). Consistent with this, we found that the LPS-induced generation of intracellular ROS was markedly abolished by pre-treatment with TSWG in the RAW 264.7 cells (Fig. 6A). Our findings revealed that TSWG increased Nrf2 activation and HO-1 expression, which may lead to the prevention of oxidative stress, as well as the supportive negative regulation of pro-inflammatory molecules in LPS-stimulated RAW 264.7 cells.

In conclusion, the present data demonstrate that TSWG possesses anti-inflammatory properties, such as the suppression of NO, TNF- $\alpha$ and IL- $1 \beta$ production through the downregulation of their corresponding gene expression in LPS-stimulated RAW 264.7 macrophages. TSWG also significantly inhibited the LPS-induced inflammation-associated signaling pathways, including the nuclear translocation of NF- $\kappa \mathrm{B}$ and the phosphorylation of Akt and MAPKs. In addition, TSWG suppressed the increased generation of intracellular ROS in LPS-stimulated RAW 264.7 cells and promoted HO-1 protein expression by promoting Nrf2 activation. Although further studies are required to clearly elucidate the exact mechanisms through which TSWG inhibits oxidative stress and the possible crosstalk between the NF- $\kappa \mathrm{B}$ and Nrf2/HO-1 signaling pathways, this study may provide a molecular basis for the therapeutic use of TSWG against various inflammatory diseases. 


\section{Acknowledgements}

This study was supported by the National Research Foundation of Korea (NRF) grant funded by the Korea government (MSIP) (no. NRF-2014R1A2A1A09006983) and the Blue-Bio Industry Regional Innovation Center (RIC08-06-07) at Dongeui University as a RIC program under the Ministry of Trade, Industry and Energy and Busan city.

\section{References}

1. Zhang X and Mosser DM: Macrophage activation by endogenous danger signals. J Pathol 214: 161-178, 2008.

2. Cinel I and Opal SM: Molecular biology of inflammation and sepsis: a primer. Crit Care Med 37: 291-304, 2009.

3. Guha M and Mackman N: LPS induction of gene expression in human monocytes. Cell Signal 13: 85-94, 2001.

4. Korbecki J, Baranowska-Bosiacka I, Gutowska I and Chlubek D: The effect of reactive oxygen species on the synthesis of prostanoids from arachidonic acid. J Physiol Pharmacol 64: 409-421, 2013.

5. Ito K: Impact of post-translational modifications of proteins on the inflammatory process. Biochem Soc Trans 35: 281-283, 2007.

6. Ivanenkov YA, Balakin KV and Tkachenko SE: New approaches to the treatment of inflammatory disease: focus on smallmolecule inhibitors of signal transduction pathways. Drugs R D 9: 397-434, 2008.

7. Geronikaki AA and Gavalas AM: Antioxidants and inflammatory disease: synthetic and natural antioxidants with anti-inflammatory activity. Comb Chem High Throughput Screen 9: 425-442, 2006.

8. Kaspar JW, Niture SK and Jaiswal AK: Nrf2:INrf2 (Keap1) signaling in oxidative stress. Free Radic Biol Med 47: 1304-1309, 2009.

9. Lee HS, Lee GS, Kim SH, Kim HK, Suk DH and Lee DS: Antioxidizing effect of the dichloromethane and hexane fractions from Orostachys japonicus in LPS-stimulated RAW 264.7 cells via upregulation of Nrf2 expression and activation of MAPK signaling pathway. BMB Rep 47: 98-103, 2014.

10. Zhao CR, Gao ZH and Qu XJ: Nrf2-ARE signaling pathway and natural products for cancer chemoprevention. Cancer Epidemiol 34: 523-533, 2010

11. Paine A, Eiz-Vesper B, Blasczyk R and Immenschuh S: Signaling to heme oxygenase-1 and its anti-inflammatory therapeutic potential. Biochem Pharmacol 80: 1895-1903, 2010.

12. Su ZY, Shu L, Khor TO, Lee JH, Fuentes F and Kong AN: A perspective on dietary phytochemicals and cancer chemoprevention: oxidative stress, nrf2, and epigenomics. Top Curr Chem 329: 133-162, 2013.

13. Zhang D, Yasuda T, Yu Y, Zheng P, Kawabata T, Ma Y and Okada S: Ginseng extract scavenges hydroxyl radical and protects unsaturated fatty acids from decomposition caused by iron-mediated lipid peroxidation. Free Radic Biol Med 20: 145-150, 1996.

14. Yun TK: Experimental and epidemiological evidence on non-organ specific cancer preventive effect of Korean ginseng and identification of active compounds. Mutat Res 523-524: 63-74, 2003.

15. Yun TK: Experimental and epidemiological evidence of the cancer-preventive effects of Panax ginseng C.A. Meyer. Nutr Rev 54: S71-S81, 1996.

16. Dey L, Xie JT, Wang A, Wu J, Maleckar SA and Yuan CS: Antihyperglycemic effects of ginseng: comparison between root and berry. Phytomedicine 10: 600-605, 2003.

17. Attele AS, Zhou YP, Xie JT, Wu JA, Zhang L, Dey L, Pugh W, Rue PA, Polonsky KS and Yuan CS: Antidiabetic effects of Panax ginseng berry extract and the identification of an effective component. Diabetes 51: 1851-1858, 2002.

18. Liu ZQ, Luo XY, Liu GZ, Chen YP, Wang ZC and Sun YX: In vitro study of the relationship between the structure of ginsenoside and its antioxidative or prooxidative activity in free radical induced hemolysis of human erythrocytes. J Agric Food Chem 51: 2555-2558, 2003.

19. Xie JT, McHendale S and Yuan CS: Ginseng and diabetes. Am J Chin Med 33: 397-404, 2005.
20. Nag SA, Qin JJ, Wang W, Wang MH, Wang H and Zhang R: Ginsenosides as anticancer agents: In vitro and in vivo activities, structure-activity relationships, and molecular mechanisms of action. Front Pharmacol 3: 25, 2012.

21. Kim JH: Cardiovascular diseases and Panax ginseng: A review on molecular mechanisms and medical applications. J Ginseng Res 36: 16-26, 2012.

22. Shibata S: Chemistry and cancer preventing activities of ginseng saponins and some related triterpenoid compounds. J Korean Med Sci 16 (Suppl): S28-S37, 2001.

23. Lee B, Park J, Kwon S, Park MW, Oh SM, Yeom MJ, Shim I, Lee HJ and Hahm DH: Effect of wild ginseng on scopolamineinduced acetylcholine depletion in the rat hippocampus. J Pharm Pharmacol 62: 263-271, 2010.

24. Mizuno M, Yamada J, Terai H, Kozukue N, Lee YS and Tsuchida H: Differences in immunomodulating effects between wild and cultured Panax ginseng. Biochem Biophys Res Commun 200: 1672-1678, 1994.

25. Park BG, Jung HJ, Cho YW, Lim HW and Lim CJ: Potentiation of antioxidative and anti-inflammatory properties of cultured wild ginseng root extract through probiotic fermentation. J Pharm Pharmacol 65: 457-464, 2013.

26. Lee B, Kim H, Shim I, Lee H and Hahm DH: Wild ginseng attenuates anxiety- and depression-like behaviors during morphine withdrawal. J Microbiol Biotechnol 21: 1088-1096, 2011.

27. Park JS, Hwang SY, Lee WS, Yu KW, Paek KY, Hwang BY and Han K: The therapeutic effect of tissue cultured root of wild Panax ginseng C.A. Mayer on spermatogenetic disorder. Arch Pharm Res 29: 800-807, 2006.

28. Li H, Lee JH and Ha JM: Effective purification of ginsenosides from cultured wild ginseng roots,red ginseng, and white ginseng with macroporous resins. J Microbiol Biotechnol 18: 1789-1791, 2008.

29. Song JJ, Lim HW, Kim K, Kim KM, Cho S and Chae SW: Effect of caffeic acid phenethyl ester (CAPE) on $\mathrm{H}_{2} \mathrm{O}_{2}$ induced oxidative and inflammatory responses in human middle ear epithelial cells. Int J Pediatr Otorhinolaryngol 76: 675-679, 2012.

30. Lu YC, Yeh WC and Ohashi PS: LPS/TLR4 signal transduction pathway. Cytokine 42: 145-151, 2008.

31. Lin YC, Huang DY, Chu CL and Lin WW: Anti-inflammatory actions of Syk inhibitors in macrophages involve non-specific inhibition of toll-like receptors-mediated JNK signaling pathway. Mol Immunol 47: 1569-1578, 2010.

32. Rietschel ET, Kirikae T, Schade FU, Ulmer AJ, Holst O, Brade H, Schmidt G, Mamat U, Grimmecke HD, Kusumoto S and Zähringer U: The chemical structure of bacterial endotoxin in relation to bioactivity. Immunobiology 187: 169-190, 1993.

33. Takeda K, Kaisho T and Akira S: Toll-like receptors. Annu Rev Immunol 21: 335-376, 2003.

34. West TE, Ernst RK, Jansson-Hutson MJ and Skerrett SJ: Activation of Toll-like receptors by Burkholderia pseudomallei. BMC Immunol 9: 46, 2008.

35. Janeway CA Jr and Medzhitov R: Lipoproteins take their toll on the host. Curr Biol 9: R879-R882, 1999.

36. Glushkova OV, Parfenyuk SB, Khrenov MO, Novoselova TV, Lunin SM, Fesenko EE and Novoselova EG: Inhibitors of TLR-4, NF- $\kappa \mathrm{B}$, and SAPK/JNK signaling reduce the toxic effect of lipopolysaccharide on RAW 264.7 cells. J Immunotoxicol 10: 133-140, 2013.

37. Kobayashi Y: The regulatory role of nitric oxide in proinflammatory cytokine expression during the induction and resolution of inflammation. J Leukoc Biol 88: 1157-1162, 2010.

38. Haddad JJ and Land SC: A non-hypoxic, ROS-sensitive pathway mediates TNF-alpha-dependent regulation of HIF-1alpha. FEBS Lett 505: 269-274, 2001.

39. Ryan KA, Smith MF Jr, Sanders MK and Ernst PB: Reactive oxygen and nitrogen species differentially regulate Toll-like receptor 4-mediated activation of NF-kappa B and interleukin-8 expression. Infect Immun 72: 2123-2130, 2004.

40. Doyle SL and O'Neill LA: Toll-like receptors: from the discovery of NFkappaB to new insights into transcriptional regulations in innate immunity. Biochem Pharmacol 72: 1102-1113, 2006.

41. Kawai T and Akira S: Signaling to NF-kappaB by Toll-like receptors. Trends Mol Med 13: 460-469, 2007.

42. Kim BH, Oh I, Kim JH, Jeon JE, Jeon B, Shin J and Kim TY: Antiinflammatory activity of compounds isolated from Astragalus sinicus L. in cytokine-induced keratinocytes and skin. Exp Mol Med 46: e87, 2014. 